\title{
GLOBAL ATTRACTOR FOR THE LATTICE DYNAMICAL SYSTEM OF A NONLINEAR BOUSSINESQ EQUATION
}

\author{
AHMED Y. ABDALLAH
}

Received 28 April 2004

We will study the lattice dynamical system of a nonlinear Boussinesq equation. Our objective is to explore the existence of the global attractor for the solution semiflow of the introduced lattice system and to investigate its upper semicontinuity with respect to a sequence of finite-dimensional approximate systems. As far as we are aware, our result here is the first concerning the lattice dynamical system corresponding to a differential equation of second order in time variable and fourth order in spatial variable with nonlinearity involving the gradients.

\section{Introduction}

Lattice dynamical systems arise naturally in many applied sciences, for instance, chemical reaction theory, material science, biology, laser systems, electrical engineering, etc. Specifically, lattice systems appear in models for propagation of pulses in myelinated axons where the membrane is excitable only at spatially discrete sites, and in this field we find much of the early theoretical development, see for example $[5,6,12,13,14,15]$. In each field they have their own forms, but in some other cases, they appear as spatial discretizations of partial differential equations. In recent years, more deep properties of the solutions of lattice dynamical systems have been studied by many researchers under various assumptions on the nonlinear part. For traveling solutions one can see $[2,3,7,8,9,20]$. The chaotic properties or pattern formation properties of solutions for such systems have been investigated in $[1,7,8,10,11,16]$.

Bates et al. [4] proved the existence of the global attractor for first-order lattice dynamical systems and investigated the approximation of the attractor by the corresponding ones of finite dimensional ordinary differential equations. Zhou [18] introduced a new weight norm to show the existence of the global attractor for second order lattice dynamical systems and to study the upper semicontinuity of the attractor, the idea of his work originated from $[4,17]$. In [19] Zhou presented the general technique needed for investigating the attractors of the lattice dynamical systems, and he studied first and second order systems. 
Consider the Hilbert space,

$$
l^{2}:=\left\{u=\left(u_{i}\right)_{i \in \mathbb{Z}}, u_{i} \in \mathbb{R}: \sum_{i \in \mathbb{Z}}\left(u_{i}\right)^{2}<\infty\right\},
$$

whose inner product and norm are given by: for all $u=\left(u_{i}\right)_{i \in \mathbb{Z}}, v=\left(v_{i}\right)_{i \in \mathbb{Z}} \in l^{2}$,

$$
\langle u, v\rangle=\sum_{i \in \mathbb{Z}} u_{i} v_{i}, \quad\|u\|=\langle u, u\rangle^{1 / 2} .
$$

Define the linear operators $D, D^{*}, B$, and $A$ from $l^{2}$ into $l^{2}$ as follows: For any $u=$ $\left(u_{i}\right)_{i \in \mathbb{Z}} \in l^{2}$,

$$
\begin{gathered}
(D u)_{i}=u_{i+1}-u_{i}, \quad\left(D^{*} u\right)_{i}=u_{i}-u_{i-1}, \quad(B u)_{i}=u_{i+1}-2 u_{i}+u_{i-1}, \\
(A u)_{i}=u_{i+2}-4 u_{i+1}+6 u_{i}-4 u_{i-1}+u_{i-2}, \quad i \in \mathbb{Z} .
\end{gathered}
$$

Then we can check that all these are bounded linear operators on $l^{2}$, and we have

$$
B=D^{*} D=D D^{*}, \quad A=B^{2} .
$$

Here we investigate the asymptotic behavior of solutions for the second order lattice dynamical system:

$$
\ddot{u}_{1}+\delta \dot{u}_{i}+\alpha(A u)_{i}+\beta(B u)_{i}+\lambda u_{i}-\frac{1}{3} k\left(D\left(D^{*} u\right)^{3}\right)_{i}=f_{i}, \quad i \in \mathbb{Z},
$$

with the initial conditions

$$
u_{i}(0)=u_{i, 0}, \quad \dot{u}_{i}(0)=u_{1 i, 0}, \quad i \in \mathbb{Z},
$$

where $\alpha, \delta, \lambda$, and $k$ are positive constants, $\beta$ is a real constant, $f=\left(f_{i}\right)_{i \in \mathbb{Z}} \in l^{2}$, and

$$
\lambda>4|\beta| \text {. }
$$

Equation (1.5) can be regarded as a spatial discretization of the following damped semilinear wave equation with continuous spatial variable $x \in \mathbb{R}$, and $t \in \mathbb{R}^{+}$,

$$
u_{t t}+\delta u_{t}+\lambda u+\alpha u_{x x x x}+\beta u_{x x}-k\left(u_{x}\right)^{2} u_{x x}=f .
$$

It is clear that (1.8) represents a nonlinear Boussinesq differential equation on the unbounded domain $\Omega=(-\infty, \infty)$.

The Boussinesq equations appear in various applications of physics and mechanics, such as: long waves in shallow water, nonlinear elastic beam systems, thermomechanical phase transitions, and some Hamiltonian mechanics.

From the previous results, in general, it is difficult to estimate the attractor of the solution semiflow generated by the initial value problem of dissipative PDEs on unbounded domains because it is infinite-dimensional. Therefore it is significant to investigate the lattice dynamical systems corresponding to the initial value problem of PDEs on unbounded domains because of the importance of such systems and they can be considered as approximations to the corresponding continuous PDEs. 


\section{The existence and uniqueness of solutions}

For $u=\left(u_{i}\right)_{i \in \mathbb{Z}}, v=\left(v_{i}\right)_{i \in \mathbb{Z}} \in l^{2}$, define a bilinear form as

$$
\langle u, v\rangle_{\lambda}=\langle B u, B v\rangle+\lambda\langle u, v\rangle, \quad\|u\|_{\lambda}=\left(\|B u\|^{2}+\lambda\|u\|^{2}\right)^{1 / 2} .
$$

We can easily show that the above bilinear form $\langle\cdot, \cdot\rangle_{\lambda}$ is an inner product of the space $l^{2}$.

It is clear that for $u=\left(u_{i}\right)_{i \in \mathbb{Z}} \in l^{2}$,

$$
\lambda\|u\|^{2} \leq\|u\|_{\lambda}^{2} \leq(16+\lambda)\|u\|^{2} .
$$

Denote by $l^{2}$ and $l_{\lambda}^{2}$ the spaces with the inner products and norms, respectively,

$$
l^{2}=\left(l^{2},\langle\cdot, \cdot\rangle,\|\cdot\|\right), \quad l_{\lambda}^{2}=\left(l_{\lambda}^{2},\langle\cdot, \cdot\rangle_{\lambda},\|\cdot\|_{\lambda}\right),
$$

then $l^{2}$ and $l_{\lambda}^{2}$ are equivalent Hilbert spaces.

Consider the Hilbert space, $E=l_{\lambda}^{2} \times l^{2}$, endowed with the inner product and norm as: for $\varphi_{j}=\left(u^{(j)}, v^{(j)}\right)^{T}=\left(\left(u_{i}^{(j)}\right),\left(v_{i}^{(j)}\right)\right)_{i \in \mathbb{Z}}^{T} \in E, j=1,2$,

$$
\begin{gathered}
\left\langle\varphi_{1}, \varphi_{2}\right\rangle_{E}=\left\langle u^{(1)}, u^{(2)}\right\rangle_{\lambda}+\left\langle v^{(1)}, v^{(2)}\right\rangle, \\
\|\varphi\|_{E}=\langle\varphi, \varphi\rangle_{E}^{1 / 2}, \quad \forall \varphi \in E .
\end{gathered}
$$

We can present (1.5) as an abstract ordinary differential equation in the Hilbert space $E$. With the above notation (1.5) can be written as

$$
\ddot{u}+\delta \dot{u}+\alpha A u+\beta B u+\lambda u-\frac{1}{3} k D\left(D^{*} u\right)^{3}=f, \quad \forall t>0,
$$

and the initial data (1.6) are

$$
u(0)=\left(u_{i, 0}\right)_{i \in \mathbb{Z}}=u_{0}, \quad \dot{u}(0)=\left(u_{1 i, 0}\right)_{i \in \mathbb{Z}}=u_{10}, \quad i \in \mathbb{Z},
$$

where $u=\left(u_{i}\right)_{i \in \mathbb{Z}}, f=\left(f_{i}\right)_{i \in \mathbb{Z}}$.

Let $v=\dot{u}+\varepsilon u, \varepsilon>0$. Taking into account condition (1.7), we can choose $\varepsilon>0$ such that

$$
\frac{\varepsilon^{2}}{2}+\frac{3 \varepsilon}{2}-\delta \leq 0, \quad \varepsilon(1+\delta)+4|\beta|-\lambda \leq 0, \quad \frac{\delta}{4}-\varepsilon \geq 0
$$

then the system (2.5) and (2.6) can be written as the following initial value problem in the Hilbert space $E$,

$$
\dot{\varphi}+C(\varphi)=F(\varphi), \quad \varphi(0)=\left(u_{0}, v_{0}\right)^{T}=\left(u_{0}, u_{10}+\varepsilon u_{0}\right)^{T} \in E,
$$


where

$$
\begin{aligned}
\varphi & =(u, v)^{T}, \quad v=\dot{u}+\varepsilon u, \\
C(\varphi) & =\left(\begin{array}{c}
\varepsilon u-v \\
\alpha A u+\lambda u+(\delta-\varepsilon)(v-\varepsilon u)
\end{array}\right), \\
F(\varphi) & =(0, g(u))^{T} .
\end{aligned}
$$

Taking into account

$$
g(u)=-\beta B u+\frac{1}{3} k D\left(D^{*} u\right)^{3}+f
$$

Now, for $u=\left(u_{i}\right)_{i \in \mathbb{Z}} \in l^{2}$, it is easy to show that

$$
\left\|D^{*} u\right\|^{2}=\|D u\|^{2} \leq 4\|u\|^{2}, \quad\|B u\|^{2} \leq 16\|u\|^{2}, \quad\|A u\|^{2} \leq 288\|u\|^{2},
$$

and we have

$$
\left\|D\left(D^{*} u\right)^{3}\right\|^{2} \leq 4\left\|\left(D^{*} u\right)^{3}\right\|^{2} \leq 4\left\|D^{*} u\right\|^{6} \leq 256\|u\|^{6} .
$$

From (2.13) and (2.14), it is clear that if $u=\left(u_{i}\right)_{i \in \mathbb{Z}} \in l^{2}$, then $A u$, and $g(u)$ are both in $l^{2}$, and in such a case for $\varphi=(u, v)^{T} \in E$ we have $C(\varphi)$ and $F(\varphi)$, given by (2.10) and (2.11), map $E$ into $E$.

Here we prove that $F$, given by (2.11), is locally Lipschitz from $E$ into $E$. Let $\varphi_{j}=$ $\left(u^{(j)}, v^{(j)}\right)^{T}=\left(\left(u_{i}^{(j)}\right),\left(v_{i}^{(j)}\right)\right)_{i \in \mathbb{Z}}^{T} \in G, j=1,2$, where $G$ is a bounded set in $E$. Using (2.13), it follows that

$$
\begin{aligned}
\left\|\left(F \varphi_{1}\right)-F\left(\varphi_{2}\right)\right\|_{E}^{2} & =\left\|g\left(u^{(1)}\right)-g\left(u^{(2)}\right)\right\|^{2} \\
& \leq 2 \beta^{2}\left\|B\left(u^{(1)}-u^{(2)}\right)\right\|^{2}+\frac{2}{9} k^{2}\left\|D\left(\left(D^{*} u^{(1)}\right)^{3}-\left(D^{*} u^{(2)}\right)^{3}\right)\right\|^{2} \\
& \leq 2 \beta^{2}\left\|B\left(u^{(1)}-u^{(2)}\right)\right\|^{2}+\frac{8}{9} k^{2}\left\|\left(D^{*} u^{(1)}\right)^{3}-\left(D^{*} u^{(2)}\right)^{3}\right\|^{2} .
\end{aligned}
$$

But

$$
\begin{aligned}
\left\|\left(D^{*} u^{(1)}\right)^{3}-\left(D^{*} u^{(2)}\right)^{3}\right\|^{2} & \leq \frac{9}{4}\left\|D^{*} u^{(1)}-D^{*} u^{(2)}\right\|^{2}\left\|\left(D^{*} u^{(1)}\right)^{2}+\left(D^{*} u^{(2)}\right)^{2}\right\|^{2} \\
& \leq \frac{9}{4}\left\|D^{*}\left(u^{(1)}-u^{(2)}\right)\right\|^{2}\left(\left\|D^{*} u^{(1)}\right\|^{2}+\left\|D^{*} u^{(2)}\right\|^{2}\right)^{2} \\
& \leq 576\left\|u^{(1)}-u^{(2)}\right\|^{2}\left(\left\|u^{(1)}\right\|^{2}+\left\|u^{(2)}\right\|^{2}\right)^{2} .
\end{aligned}
$$


Because $G$ is a bounded set in $E$, there exists a constant $L_{1}=L_{1}(G)>0$ such that

$$
\left\|\left(D^{*}\left(u^{(1)}\right)\right)^{3}-\left(D^{*}\left(u^{(2)}\right)\right)^{3}\right\|^{2} \leq L_{1}\left\|u^{(1)}-u^{(2)}\right\|^{2} .
$$

If we substitute (2.17) into (2.15), and if we let

$$
L_{2}=\max \left\{2 \beta^{2}, \frac{8}{9} k^{2} L_{1} \lambda^{-1}\right\}
$$

then we get

$$
\begin{aligned}
\left\|F\left(\varphi_{1}\right)-F\left(\varphi_{2}\right)\right\|_{E}^{2} & \leq L_{2}\left(\left\|B\left(u^{(1)}-u^{(2)}\right)\right\|^{2}+\lambda\left\|u_{i}^{(1)}-u_{i}^{(2)}\right\|^{2}\right) \\
& \leq L_{2}\left\|\varphi_{1}-\varphi_{2}\right\|_{E}^{2}
\end{aligned}
$$

That $F$ is locally Lipschitz from $E$ into $E$. It is easy to see that $C$, given by (2.10), is locally Lipschitz from $E$ into $E$ since $A$ is a bounded linear operator from $l^{2}$ into $l^{2}$. Therefore using the standard theory of ordinary differential equations, we find that there exists a unique local solution $\varphi$ for the initial value problem (2.8). That we have the following lemma.

Lemma 2.1. For any initial data $\varphi(0)=\left(u_{0}, v_{0}\right)^{T} \in E$, there exists a unique local solution $\varphi(t)=(u(t), v(t))^{T}$ of the initial value problem (2.8), such that $\varphi \in C^{1}([0, T), E)$, for some $T>0$. If $T<\infty$, then $\lim _{t \rightarrow T^{-}}\|\varphi\|_{E}^{2}=\infty$.

It is shown in Lemma 3.1, below, that the solution $\varphi(t)$ of $(2.8)$ exists globally, that is $\varphi \in C^{1}([0, \infty), E)$, which implies that the maps from $E$ into $E$,

$$
S_{\mathcal{\varepsilon}}(t): \varphi(0)=\left(u_{0}, v_{0}\right)^{T} \longrightarrow \varphi(t)=S_{\mathcal{\varepsilon}}(t) \varphi(0)=(u(t), v(t))^{T}, \quad t \geq 0,
$$

generate a continuous semigroup $\left\{S_{\varepsilon}(t)\right\}_{t \geq 0}$ on $E$, where $v(t)=\dot{u}(t)+\varepsilon \mathcal{u}(t)$. We can also call it the solution semiflow of (2.8).

\section{The boundedness of global solutions}

First, note that for all $u=\left(u_{i}\right)_{i \in \mathbb{Z},} v=\left(v_{i}\right)_{i \in \mathbb{Z}} \in l^{2}$, we have

$$
\langle D u, v\rangle=-\left\langle u, D^{*} v\right\rangle, \quad\langle B u, v\rangle=-\langle D u, D v\rangle, \quad\langle A u, v\rangle=\langle B u, B v\rangle .
$$

Let $\varphi(t)=(u(t), v(t))^{T} \in E$ be a solution of (2.8), where again $v(t)=\dot{u}(t)+\varepsilon u(t)$, and take the inner product $\langle\cdot, \cdot\rangle_{E}$ of $(2.8)$ with $\varphi(t)$, we obtain

$$
\left\langle\ddot{u}+\delta \dot{u}+\alpha A u+\beta B u+\lambda u-\frac{1}{3} k D\left(D^{*} u\right)^{3}-f, \dot{u}+\varepsilon u\right\rangle=0 .
$$


660

Taking into account (3.1) and the following results:

$$
\begin{gathered}
-\frac{1}{3} k\left\langle D\left(\left(D^{*} u\right)^{3}\right), \dot{u}\right\rangle=\frac{1}{3} k\left\langle\left(D^{*} u\right)^{3}, D^{*} \dot{u}\right\rangle=\frac{1}{12} k \frac{d}{d t}\left\|\left(D^{*} u\right)^{2}\right\|^{2}, \\
\varepsilon\langle\ddot{u}, u\rangle=\varepsilon\left(\frac{d}{d t}\langle\dot{u}, u\rangle-\|\dot{u}\|^{2}\right) .
\end{gathered}
$$

We can write (3.2) in the following form:

$$
\frac{d}{d t} P(t)+N(t)=0
$$

where

$$
\begin{aligned}
P(t)= & \frac{1}{2}\|\dot{u}\|^{2}+\frac{\alpha}{2}\|B u\|^{2}-\frac{\beta}{2}\|D u\|^{2}+\frac{\lambda}{2}\|u\|^{2} \\
& +\frac{1}{12} k\left\|\left(D^{*} u\right)^{2}\right\|^{2}-\langle f, u\rangle+\varepsilon\langle\dot{u}, u\rangle+\frac{\varepsilon \delta}{2}\|u\|^{2}, \\
N(t)=(\delta-\varepsilon)\|\dot{u}\|^{2}+ & \varepsilon \alpha\|B u\|^{2}-\varepsilon \beta\|D u\|^{2}+\varepsilon \lambda\|u\|^{2}+\frac{\varepsilon}{3} k\left\|\left(D^{*} u\right)^{2}\right\|^{2}-\varepsilon\langle f, u\rangle .
\end{aligned}
$$

Now

$$
\begin{aligned}
\varepsilon P(t)-N(t)= & \left(\frac{3 \varepsilon}{2}-\delta\right)\|\dot{u}\|^{2}-\frac{\varepsilon \alpha}{2}\|B u\|^{2}+\frac{\varepsilon \beta}{2}\|D u\|^{2}+\frac{\varepsilon}{2}(\varepsilon \delta-\lambda)\|u\|^{2} \\
& -\frac{\varepsilon}{4} k\left\|\left(D^{*} u\right)^{2}\right\|^{2}+\varepsilon^{2}\langle\dot{u}, u\rangle .
\end{aligned}
$$

Since

$$
\begin{gathered}
\frac{\varepsilon \beta}{2}\|D u\|^{2} \leq \frac{\varepsilon|\beta|}{2}\|D u\|^{2} \leq 2 \varepsilon|\beta|\|u\|^{2}, \\
\varepsilon^{2}\langle\dot{u}, u\rangle \leq \frac{\varepsilon^{2}}{2}\|\dot{u}\|^{2}+\frac{\varepsilon^{2}}{2}\|u\|^{2} .
\end{gathered}
$$

It is easy to see that

$$
\varepsilon P(t)-N(t) \leq\left(\frac{\varepsilon^{2}}{2}+\frac{3 \varepsilon}{2}-\delta\right)\|\dot{u}\|^{2}+\frac{\varepsilon}{2}(\varepsilon(1+\delta)+4|\beta|-\lambda)\|u\|^{2} .
$$

From (2.7), it follows that

$$
\varepsilon P(t)-N(t) \leq 0
$$

From (3.4) and (3.9), it follows that for $t>0$,

$$
\frac{d}{d t} P(t)+\varepsilon P(t) \leq 0 .
$$


By using the Gronwall lemma, it follows that

$$
P(t) \leq P(0) e^{-\varepsilon t}
$$

But we know that

$$
\begin{gathered}
\varepsilon\langle\dot{u}, u\rangle \geq-\frac{1}{4}\|\dot{u}\|^{2}-\varepsilon^{2}\|u\|^{2}, \\
-\frac{\beta}{2}\|D u\|^{2} \geq-\frac{|\beta|}{2}\|D u\|^{2} \geq-2|\beta|\|u\|^{2},
\end{gathered}
$$

and for any $M_{1}>0$, we have

$$
-\langle f, u\rangle \geq-\frac{2}{M_{1}}\|f\|^{2}-\frac{M_{1}}{8}\|u\|^{2}
$$

From (3.5), we get

$$
P(t) \geq \frac{1}{4}\|\dot{u}\|^{2}+\frac{\alpha}{2}\|B u\|^{2}+\left(\frac{\lambda}{2}+\frac{\varepsilon \delta}{2}-\varepsilon^{2}-\frac{M_{1}}{8}-2|\beta|\right)\|u\|^{2}-\frac{2}{M_{1}}\|f\|^{2} .
$$

From (1.7), we know that $\lambda>4|\beta|$, and we can choose $M_{1}$ as small as desired such that

$$
\lambda\left(1-\frac{M_{1}}{4 \lambda}-2 M_{1}\right)-4|\beta| \geq 0
$$

and in such a case, we can write (3.14) in the following form:

$$
\begin{aligned}
P(t) \geq & \frac{1}{4}\|\dot{u}\|^{2}+\frac{\alpha}{2}\|B u\|^{2}+\lambda M_{1}\|u\|^{2} \\
& +\left(\lambda\left(\frac{1}{2}-\frac{M_{1}}{8 \lambda}-M_{1}\right)+\frac{\varepsilon \delta}{2}-\varepsilon^{2}-2|\beta|\right)\|u\|^{2}-\frac{2}{M_{1}}\|f\|^{2} \\
\geq & \frac{1}{4}\|\dot{u}\|^{2}+\frac{\varepsilon \delta}{4}\|u\|^{2}+\frac{\alpha}{2}\|B u\|^{2}+\lambda M_{1}\|u\|^{2}-\frac{2}{M_{1}}\|f\|^{2},
\end{aligned}
$$

because $\varepsilon \delta / 4-\varepsilon^{2} \geq 0$ from (2.7). If we choose

$$
M_{2}=\min \left\{\frac{1}{8}, \frac{\delta}{8 \varepsilon}, \frac{\alpha}{2}, M_{1}\right\}
$$

then it follows that

$$
\|B u\|^{2}+\lambda\|u\|^{2}+2\|\dot{u}\|^{2}+2 \varepsilon^{2}\|u\|^{2} \leq \frac{1}{M_{2}}\left(P(t)+\frac{2}{M_{1}}\|f\|^{2}\right),
$$


that is,

$$
\|\varphi\|_{E}^{2}=\|B u\|^{2}+\lambda\|u\|^{2}+\|v\|^{2}=\|B u\|^{2}+\lambda\|u\|^{2}+\|\dot{u}+\varepsilon u\|^{2} \leq \frac{1}{M_{2}}\left(P(t)+\frac{2}{M_{1}}\|f\|^{2}\right) .
$$

Now, from (3.11) we obtain that for $t>0$ :

$$
\begin{array}{r}
\|\varphi\|_{E}^{2} \leq \frac{1}{M_{2}}\left(P(0) e^{-\varepsilon t}+\frac{2}{M_{1}}\|f\|^{2}\right), \\
\lim _{t \rightarrow \infty}\|\varphi\|_{E}^{2} \leq \frac{2}{M_{3}}\|f\|^{2}
\end{array}
$$

where $M_{3}=M_{1} M_{2}$. Inequality (3.21) implies that the solution semigroup $\left\{S_{\varepsilon}(t)\right\}_{t \geq 0}$ of (2.8) possesses a bounded absorbing set in E. Taking into account Lemma 2.1, we have the following lemma.

Lemma 3.1. If $f \in l^{2},(1.7)$ and (2.7) are satisfied, then for any initial data in $E$, the solution $\varphi(t)$ of (2.8) exists globally, for all $t \geq 0$. Moreover, there exists a bounded ball $O=O_{E}\left(0, r_{0}\right)$ in $E$, centered at 0 with radius $r_{0}$, such that for every bounded set $G$ of $E$, there exists $T(G) \geq 0$ such that

$$
S_{\varepsilon}(t) G \subset O, \quad \forall t \geq T(G),
$$

where $r_{0}^{2}>\left(2 / M_{3}\right)\|f\|^{2}$. Therefore, there is a constant $T_{0} \geq 0$ depending on $O$ such that

$$
S_{\varepsilon}(t) O \subset O, \quad \forall t \geq T_{0} .
$$

\section{The existence of the global attractor}

To prove the existence of the global attractor for the solution semigroup $\left\{S_{\varepsilon}(t)\right\}_{t \geq 0}$ of (2.8), we need to prove the asymptotic compactness of $\left\{S_{\varepsilon}(t)\right\}_{t \geq 0}$. The key for proving the asymptotic compactness of semiflows on an unbounded domain, such as the lattice system here, is to establish uniform estimates on "Tail Ends" of solutions.

Lemma 4.1. Assume that $f \in l^{2}$, (1.7) and (2.7) are satisfied, and $\varphi(0)=\left(u_{0}, v_{0}\right)^{T}=$ $\left(u_{0}, u_{10+} \varepsilon u_{0}\right)^{T} \in O$, where $O$ is the bounded absorbing ball given by Lemma 3.1. Then for any $\eta>0$, there exist positive constants $T(\eta)$ and $K(\eta)$ such that the solution $\varphi(t)=(u(t), v(t))^{T}$ $=\left(\varphi_{i}\right)_{i \in \mathbb{Z}}=\left(\left(u_{i}(t)\right),\left(v_{i}(t)\right)\right)_{i \in \mathbb{Z}}^{T} \in E$ of $(2.8), v(t)=\dot{u}(t)+\varepsilon \mathcal{u}(t)$, satisfies

$$
\sum_{|i| \geq K(\eta)}\left\|\varphi_{i}(t)\right\|_{E}^{2}=\sum_{|i| \geq K(\eta)}\left(\left\|(B u(t))_{i}\right\|^{2}+\lambda\left\|u_{i}(t)\right\|^{2}+\left\|v_{i}(t)\right\|^{2}\right) \leq \eta
$$

for all $t \geq T(\eta)$. 
Proof. Consider a smooth increasing function $\theta \in C^{1}\left(\mathbb{R}^{+}, \mathbb{R}\right)$ such that

$$
\begin{gathered}
\theta(s)=0, \quad 0 \leq s<1, \\
0 \leq \theta(s) \leq 1, \quad 1 \leq s<2, \\
\theta(s)=1, \quad s \geq 2,
\end{gathered}
$$

and there exists a constant $M_{0}$ such that $\theta^{\prime}(s) \leq M_{0}, \forall s \in \mathbb{R}^{+}$.

Let $\varphi(t)=(u(t), v(t))^{T}=\left(\varphi_{i}\right)_{i \in \mathbb{Z}}=\left(\left(u_{i}(t)\right),\left(v_{i}(t)\right)\right)_{i \in \mathbb{Z}}^{T}$ be a solution of (2.8), where $v(t)=\dot{u}(t)+\varepsilon u(t)$. Let $m$ be a positive integer. Set $w_{i}=\theta(|i| / m) u_{i}, z_{i}=\theta(|i| / m) v_{i}$, and $y=(w, z)^{T}=\left(\left(w_{i}\right),\left(z_{i}\right)\right)_{i \in \mathbb{Z}}^{T}$.

Using (3.1), it follows that

$$
\begin{aligned}
-\frac{1}{3} k\left\langle D\left(D^{*}(u)\right)^{3}, z\right\rangle= & \frac{1}{3} k \sum_{i \in \mathbb{Z}}\left(D^{*} u\right)_{i}^{3}\left(D^{*} z\right)_{i} \\
= & \frac{1}{3} k \sum_{i \in \mathbb{Z}}\left(D^{*} u\right)_{i}^{3}\left(\theta\left(\frac{|i|}{m}\right)\left(D^{*} v\right)_{i}+\left(D^{*} z\right)_{i}-\theta\left(\frac{|i|}{m}\right)\left(D^{*} v\right)_{i}\right) \\
= & \frac{1}{3} k \sum_{i \in \mathbb{Z}}\left(\theta\left(\frac{|i|}{m}\right)\left(\frac{1}{4} \frac{d}{d t}\left(D^{*} u\right)_{i}^{4}+\varepsilon\left(D^{*} u\right)_{i}^{4}\right)\right. \\
& \left.+\left(D^{*} u\right)_{i}^{3}\left(\left(D^{*} z\right)_{i}-\theta\left(\frac{|i|}{m}\right)\left(D^{*} v\right)_{i}\right)\right) .
\end{aligned}
$$

Similarly, one can rewrite $\alpha\langle A u, z\rangle$ and $\beta\langle B u, z\rangle$. In such a case, considering the inner product of (2.8) with $y$, we get that

$$
\frac{d}{d t} P_{1}(t)+N_{1}(t)=0,
$$

where

$$
\begin{gathered}
P_{1}(t)=\sum_{i \in \mathbb{Z}}\left(\theta\left(\frac{|i|}{m}\right)\left(\begin{array}{c}
\frac{1}{2} \dot{u}_{i}^{2}+\frac{\alpha}{2}(B u)_{i}^{2}-\frac{\beta}{2}(D u)_{i}^{2}+\frac{\lambda}{2} u_{i}^{2} \\
+\frac{k}{12}\left(D^{*} u\right)_{i}^{4}-f_{i} u_{i}+\varepsilon \dot{u}_{i} u_{i}+\frac{\varepsilon \delta}{2} u_{i}^{2}
\end{array}\right)\right), \\
N_{1}(t)=\sum_{i \in \mathbb{Z}}\left(\theta\left(\frac{|i|}{m}\right)\left((\delta-\varepsilon) \dot{u}_{i}^{2}+\varepsilon \alpha(B u)_{i}^{2}-\varepsilon \beta(D u)_{i}^{2}+\varepsilon \lambda u_{i}^{2}+\frac{\varepsilon k}{3}\left(D^{*} u\right)_{i}^{4}-\varepsilon f_{i} u_{i}\right)\right) \\
+\sum_{i \in \mathbb{Z}}\left(\begin{array}{c}
\left.\alpha(B u)_{i}\left((B z)_{i}-\theta\left(\frac{|i|}{m}\right)(B v)_{i}\right)-\beta(D u)_{i}\left((D z)_{i}-\theta\left(\frac{|i|}{m}\right)(D v)_{i}\right)\right) . \\
+\frac{1}{3} k\left(D^{*} u\right)_{i}^{3}\left(\left(D^{*} z\right)_{i}-\theta\left(\frac{|i|}{m}\right)\left(D^{*} v\right)_{i}\right)
\end{array}\right) .
\end{gathered}
$$


Along the lines of (69) of [19], taking into account Lemma 3.1, it follows that there exists a constant $R_{1}=R_{1}\left(M_{0}, r_{0}\right)$ such that for $t \geq T_{0}$

$$
\sum_{i \in \mathbb{Z}}\left(\begin{array}{c}
\alpha(B u)_{i}\left((B z)_{i}-\theta\left(\frac{|i|}{m}\right)(B v)_{i}\right)-\beta(D u)_{i}\left((D z)_{i}-\theta\left(\frac{|i|}{m}\right)(D v)_{i}\right) \\
+\frac{1}{3} k\left(D^{*} u\right)_{i}^{3}\left(\left(D^{*} z\right)_{i}-\theta\left(\frac{|i|}{m}\right)\left(D^{*} v\right)_{i}\right)
\end{array}\right) \geq-\frac{R_{1}}{m}
$$

In such a case, if we compare $P_{1}$ and $N_{1}$ with $P$ and $N$ given within Section 3, taking into account (3.9) and (3.20), it is clear that for $t \geq T_{0}$,

$$
\begin{gathered}
\varepsilon P_{1}(t)-N_{1}(t) \leq \frac{R_{1}}{m}, \\
P_{1}(t) \leq P_{1}(0) e^{-\varepsilon t}+\frac{R_{1}}{m}\left(1-e^{-\varepsilon t}\right), \\
\sum_{i \in \mathbb{Z}} \theta\left(\frac{|i|}{m}\right)\left\|\varphi_{i}\right\|_{E}^{2} \leq \frac{1}{M_{2}}\left(P_{1}(0) e^{-\varepsilon t}+\frac{2}{M_{1}} \sum_{i \in \mathbb{Z}} \theta\left(\frac{|i|}{m}\right) f_{i}^{2}+\frac{R_{1}}{m}\right) .
\end{gathered}
$$

It is assumed that $\varphi(0) \in O$, therefore it is clear that there exists a constant $R_{2}=R_{2}\left(r_{0}\right)$ such that $P_{1}(0) \leq R_{2}$. Thus

$$
\sum_{i \in \mathbb{Z}} \theta\left(\frac{|i|}{m}\right)\left\|\varphi_{i}\right\|_{E}^{2} \leq \frac{1}{M_{2}}\left(R_{2} e^{-\varepsilon t}+\frac{2}{M_{1}} \sum_{i \in \mathbb{Z}} \theta\left(\frac{|i|}{m}\right) f_{i}^{2}+\frac{R_{1}}{m}\right), \quad \forall t \geq T_{0} .
$$

Now for $\eta>0$, there exists $T_{1}=T_{1}(\eta)$ such that

$$
\frac{1}{M_{2}} R_{2} e^{-\varepsilon t} \leq \frac{\eta}{2}
$$

and since $f \in l^{2}$, let us fix $m$ to be sufficiently large such that for $t \geq T_{0}$,

$$
\frac{1}{M_{2}}\left(\frac{2}{M_{1}} \sum_{i \in \mathbb{Z}} \theta\left(\frac{|i|}{m}\right) f_{i}^{2}+\frac{R_{1}}{m}\right) \leq \frac{1}{M_{2}}\left(\frac{2}{M_{1}} \sum_{|i| \geq m} f_{i}^{2}+\frac{R_{1}}{m}\right) \leq \frac{\eta}{2} .
$$

Let $T(\eta)=\max \left\{T_{0}, T_{1}\right\}$ and $K(\eta)=2 m$, then from (4.8), (4.9), and (4.10) it is clear that for all $t \geq T(\eta)$,

$$
\sum_{|i| \geq K(\eta)}\left\|\varphi_{i}\right\|_{E}^{2}=\sum_{|i| \geq K(\eta)}\left(\theta\left(\frac{|i|}{m}\right)\left\|\varphi_{i}\right\|_{E}^{2}\right) \leq \sum_{i \in \mathbb{Z}}\left(\theta\left(\frac{|i|}{m}\right)\left\|\varphi_{i}\right\|_{E}^{2}\right) \leq \eta .
$$

The proof is completed.

Lemma 4.2. Assume that $f \in l^{2},(1.7)$ and (2.7) are satisfied. Then the solution semigroup $\left\{S_{\mathcal{E}}(t)\right\}_{t \geq 0}$ of (2.8) is asymptotically compact in $E$, that is, if $\left\{\varphi_{n}\right\}$ is bounded in $E$ and $t_{n} \rightarrow$ $\infty$, then $\left\{S_{\mathcal{\varepsilon}}\left(t_{n}\right) \varphi_{n}\right\}$ is precompact in $E$. 
Proof. By using Lemmas 3.1 and 4.1, above, the proof of this lemma will be similar to that of [18, Lemma 3.2].

Now we are in a position to state the existence of the global attractor for the solution semigroup $\left\{S_{\varepsilon}(t)\right\}_{t \geq 0}$ of (2.8).

Theorem 4.3. If $f \in l^{2},(1.7)$, and (2.7) are satisfied, then the solution semigroup $\left\{S_{\varepsilon}(t)\right\}_{t \geq 0}$ of (2.8) possesses a global attractor $\mathscr{B}$ in $E$.

Proof. From the existence theorem of global attractors, Lemmas 3.1, and 4.2 we get the result.

If we consider the mapping

$$
S(t):\left(u_{0}, u_{10}\right)^{T} \longrightarrow(u(t), \dot{u}(t))^{T} \in l^{2} \times l^{2},
$$

which is associated with the original problem (1.5) and (1.6) in the space $l^{2} \times l^{2}$, then

$$
S(t)=R_{-\varepsilon} S_{\varepsilon}(t) R_{\varepsilon}, \quad \text { where } R_{\varepsilon}=\left(\begin{array}{ll}
1 & 0 \\
\varepsilon & 1
\end{array}\right)
$$

is an isomorphism on $l^{2} \times l^{2}$. Since the semiflow $\left\{S_{\varepsilon}(t)\right\}_{t \geq 0}$ possesses a global attractor $\mathscr{B}$ in $E$, the global attractor of $\{S(t)\}_{t \geq 0}$, in $E$, exists as $\mathscr{A}=R_{-\varepsilon} \mathscr{B}$.

\section{Upper semicontinuity of the global attractor}

Finally in this section we will consider the approximation to the global attractor $\mathscr{B}$ of the solution semigroup $\left\{S_{\varepsilon}(t)\right\}_{t \geq 0}$ by the global attractors of finite-dimensional ordinary differential systems.

Let $n \geq 2$ be an integer,

$$
\mathbb{Z}_{n}=\{i \in \mathbb{Z}:|i| \leq n\}
$$

and $w=\left(w_{i}\right)_{|i| \leq n} \in \mathbb{R}^{2 n+1}$. We consider the $(2 n+1)$-dimensional ordinary differential equations with initial data in $\mathbb{R}^{2 n+1}$ :

$$
\ddot{w}_{i}+\delta \dot{w}_{i}+\alpha(A w)_{i}+\beta(B w)_{i}+\lambda w_{i}-\frac{1}{3} k\left(D\left(D^{*} w\right)^{3}\right)_{i}=f_{i}, \quad t>0, \quad i \in \mathbb{Z}_{n}
$$

with the initial values

$$
w_{i}(0)=w_{i 0}, \quad \dot{w}_{i}(0)=z_{i 0}, \quad i \in \mathbb{Z}_{n} .
$$

Then (5.2) and (5.3) can be written as a form of vectors in $\mathbb{R}^{2 n+1}$,

$$
\begin{gathered}
\ddot{w}+\delta \dot{w}+\alpha \tilde{A} w+\beta \widetilde{B} w+\lambda w-\frac{1}{3} k \tilde{h}(w)=\tilde{f}, \\
w(0)=\left(w_{i 0}\right)_{|i| \leq n}, \quad \dot{w}(0)=\left(z_{i 0}\right)_{|i| \leq n},
\end{gathered}
$$




$$
\begin{aligned}
& \text { where } w=\left(w_{i}\right)_{|i| \leq n}, \tilde{f}=\left(f_{i}\right)_{|i| \leq n}, \tilde{h}(w)=\left(\left(D\left(D^{*} w\right)^{3}\right)_{i}\right)_{|i| \leq n} \text {, } \\
& w_{-n+1}=w_{n+2}, \quad w_{-n}=w_{n+1}, \quad w_{n}=w_{-n-1}, \quad w_{n-1}=w_{-n-2}, \\
& (\tilde{D} u)_{i}=u_{i+1}-u_{i}, \quad\left(\tilde{D}^{*} u\right)_{i}=u_{i}-u_{i-1}, \quad(\widetilde{B} u)_{i}=u_{i+1}-2 u_{i}+u_{i-1} \text {, } \\
& (\tilde{A} u)_{i}=u_{i+2}-4 u_{i+1}+6 u_{i}-4 u_{i-1}+u_{i-2}, \quad i \in \mathbb{Z}_{n},
\end{aligned}
$$

then it is clear that

$$
\widetilde{A}=\widetilde{B}^{2}, \quad \widetilde{B}=\widetilde{D}^{*} \widetilde{D}=\tilde{D} \tilde{D}^{*}
$$

For any $w=\left(w_{i}\right)_{|i| \leq n}, z=\left(z_{i}\right)_{|i| \leq n} \in \mathbb{R}^{2 n+1}$, define

$$
\begin{gathered}
\langle w, z\rangle_{\mathbb{R}^{2 n+1}}=\sum_{|i| \leq n} w_{i} z_{i}, \quad\|w\|_{\mathbb{R}^{2 n+1}}=\langle w, w\rangle_{\mathbb{R}^{2 n+1}}^{1 / 2}, \\
\langle w, z\rangle_{\mathbb{R}_{\lambda}^{2 n+1}}=\langle\widetilde{B} w, \widetilde{B} z\rangle_{\mathbb{R}^{2 n+1}}+\lambda\langle w, z\rangle_{\mathbb{R}^{2 n+1}}, \quad\|w\|_{\mathbb{R}_{\lambda}^{2 n+1}}=\langle w, z\rangle_{\mathbb{R}_{\lambda}^{2 n+1}}^{1 / 2} .
\end{gathered}
$$

In such a case it is clear that $\mathbb{R}^{2 n+1}=\left(\mathbb{R}^{2 n+1},\|\cdot\|\right)$ and $\mathbb{R}_{\lambda}^{2 n+1}=\left(\mathbb{R}^{2 n+1},\|\cdot\| \lambda\right)$ are Hilbert spaces.

Let $\widetilde{E}=\mathbb{R}_{\lambda}^{2 n+1} \times \mathbb{R}^{2 n+1}$, endowed with the inner product and norm as follows: for $W_{j}=$ $\left(w^{(j)}, z^{(j)}\right)^{T}=\left(\left(w_{i}^{(j)}\right),\left(z_{i}^{(j)}\right)\right)_{|i| \leq n}^{T} \in \widetilde{E}, j=1,2$,

$$
\left\langle W_{1}, W_{2}\right\rangle_{\widetilde{E}}=\left\langle w^{(1)}, w^{(2)}\right\rangle_{\mathbb{R}_{\lambda}^{2 n+1}}+\left\langle z^{(1)}, z^{(2)}\right\rangle_{\mathbb{R}^{2 n+1}}, \quad\left\|W_{1}\right\|_{\widetilde{E}}=\left\langle W_{1}, W_{1}\right\rangle_{\widetilde{E}}^{1 / 2}
$$

then $\widetilde{E}$ is a Hilbert space.

Let $z=\dot{w}+\varepsilon w$, where $\varepsilon>0$ satisfies (2.7). It is easy to check that problem (5.4) can be formulated to the following first-order system in the Hilbert space $\widetilde{E}$,

$$
\dot{Y}+\widetilde{C}(Y)=\widetilde{F}(Y), \quad Y(0)=(w(0), \dot{w}(0)+\varepsilon w(0))^{T} \in \widetilde{E},
$$

where $Y=(w, z)^{T}$,

$$
\begin{gathered}
\tilde{C}(Y)=\left(\begin{array}{c}
\varepsilon w-z \\
\alpha \tilde{A} w+\lambda w+(\delta-\varepsilon)(z-\varepsilon w)
\end{array}\right), \quad \tilde{F}(Y)=\left(\begin{array}{c}
0 \\
\tilde{g}(w)
\end{array}\right), \\
\tilde{g}(w)=-\beta \widetilde{B} w+\frac{1}{3} k \tilde{h}(w)+\tilde{f} .
\end{gathered}
$$

Obviously, the problems (5.4) and (5.9) are well posed in $\widetilde{E}$. From Lemma 5.1 below, the solution $Y(t)$ of (5.9) is bounded in finite time, thus, $Y(t)$ exists globally, that is, for any $Y(0) \in \widetilde{E}$, there exists a unique solution $Y \in C([0, \infty), \widetilde{E}) \cap C^{1}((0, \infty), \widetilde{E})$, and maps of solutions $S_{\varepsilon, n}(t): Y(0) \rightarrow Y(t)=S_{\varepsilon, n}(t) Y(0) \in \widetilde{E}$ generate a continuous semi$\operatorname{group}\left\{S_{\varepsilon, n}(t)\right\}_{t \geq 0}$ on $\widetilde{E}$. 
Similar to Lemma 3.1 and Theorem 4.3 we can prove the following lemma.

Lemma 5.1. If $f \in l^{2},(1.7)$ and (2.7) are satisfied, then there exists a bounded ball $\widetilde{O}=$ $\widetilde{O}_{\widetilde{E}}\left(0, r_{0}\right)$ in $\widetilde{E}$, centered at 0 with radius $r_{0}$, such that for every bounded set $\widetilde{G}$ of $\widetilde{E}$, there exists $T(\tilde{G}) \geq 0$ such that

$$
S_{\varepsilon, n}(t) \widetilde{G} \subset \widetilde{O}, \quad \forall t \geq T(\widetilde{G}), n=1,2, \ldots,
$$

where $r_{0}$ is the same constant given by Lemma 3.1, and it is independent of $n$. Moreover, the semigroup $\left\{S_{\varepsilon, n}(t)\right\}_{t \geq 0}$ possesses a global attractor $\mathscr{B}_{n}, \mathscr{B}_{n} \subset \widetilde{O} \subset \widetilde{E}$.

Here we prove that the global attractors $\mathscr{B}_{n}$ of the semigroup $\left\{S_{n}(t)\right\}_{t \geq 0}$ converge to the global attractor $\mathscr{B}$ of the semigroup $\{S(t)\}_{t \geq 0}$. In such a case we should extend the element $u=\left(u_{i}\right)_{\|i\|_{m} \leq n} \in \mathbb{R}^{2 n+1}$ to an element of $l^{2}$ such that $u_{i}=0$ for $|i|>n$, still denote it by $u$.

Lemma 5.2. If $f \in l^{2},(1.7)$ and (2.7) are satisfied, and $\varphi_{n}(0) \in \mathscr{B}_{n}$, then there exists a subsequence $\left\{\varphi_{n_{k}}(0)\right\}$ of $\left\{\varphi_{n}(0)\right\}$ and $\varphi_{0} \in \mathscr{B}$ such that $\varphi_{n_{k}}(0)$ converges to $\varphi_{0}$ in $E$.

Proof. Consider $\varphi_{n}(t)=S_{n}(t) \varphi_{n}(0)=\left(u_{n}(t), v_{n}(t)\right)^{T} \in \widetilde{E}$ to be a solution of (5.9). Since $\varphi_{n}(0) \in \mathscr{B}_{n}, \varphi_{n}(t) \in \mathscr{B}_{n} \subset \widetilde{O}$ for all $t \in \mathbb{R}^{+}$, and again the element $\varphi_{n}(t)=\left(\varphi_{n, i}(t)\right)_{|i| \leq n} \in$ $\widetilde{E}$ can be extended to an element of $E$ such that $\varphi_{n, i}(t)=(0,0)^{T}$ for $|i|>n$, still denote it by $\varphi_{n}(t)$, it follows that

$$
\left\|\varphi_{n}(t)\right\|_{\tilde{E}}=\left\|\varphi_{n}(t)\right\|_{E}=\left(\left\|B u_{n}\right\|^{2}+\lambda\left\|u_{n}\right\|^{2}+\left\|v_{n}\right\|^{2}\right)^{1 / 2} \leq r_{0}, \quad \forall t \in \mathbb{R}^{+}, n=1,2, \ldots
$$

From (2.13) and (5.12), it follows that there exists a constant $C_{1}=C_{1}\left(r_{0}\right)$ such that

$$
\begin{aligned}
\left\|\widetilde{C}\left(\varphi_{n}(t)\right)\right\|_{\widetilde{E}}^{2} & \leq\left\|C\left(\varphi_{n}(t)\right)\right\|_{E}^{2} \\
& =\left\|B\left(\varepsilon u_{n}-v_{n}\right)\right\|^{2}+\lambda\left\|\varepsilon u_{n}-v_{n}\right\|^{2}+\left\|\alpha A u_{n}+\lambda u_{n}+(\delta-\varepsilon)\left(v_{n}-\varepsilon u_{n}\right)\right\|^{2} \\
& \leq C_{1}, \quad \forall t \in \mathbb{R}^{+}, n=1,2, \ldots
\end{aligned}
$$

Similarly, we can extend the element $\tilde{f}=\left(f_{i}\right)_{|i| \leq n}$ to the element $f_{n}=\left(f_{n, i}\right)_{i \in \mathbb{Z}} \in l^{2}$ such that $f_{n, i}=f_{i}$ for $|i| \leq n$, and $f_{n, i}=0$ for $|i|>n$. In such a case, by using (2.13), (2.14), and (5.12), there exists a constant $C_{2}=C_{2}\left(r_{0},\|f\|\right)$ such that

$$
\begin{aligned}
\left\|\widetilde{F}\left(\varphi_{n}(t)\right)\right\|_{\widetilde{E}}^{2} & \leq\left\|F\left(\varphi_{n}(t)\right)\right\|_{E}^{2}=\left\|-\beta B u_{n}+\frac{1}{3} k D\left(D^{*} u_{n}\right)^{3}+f_{n}\right\|^{2} \\
& \leq C_{2}, \quad \forall t \in \mathbb{R}^{+}, n=1,2, \ldots
\end{aligned}
$$

From (5.9), we obtain that

$$
\left\|\dot{\varphi}_{n}(t)\right\|_{E}^{2} \leq 2\left\|C\left(\varphi_{n}(t)\right)\right\|_{E}^{2}+2\left\|F\left(\varphi_{n}(t)\right)\right\|_{E}^{2} .
$$


Thus by using (5.13), (5.14), and (5.15), it follows that there exists a constant $C_{3}=$ $C_{3}\left(r_{0},\|f\|\right)$ such that

$$
\left\|\dot{\varphi}_{n}(t)\right\|_{E} \leq C_{3}, \quad \forall t \in \mathbb{R}^{+}, n=1,2, \ldots
$$

Let $J_{k}(k=1,2, \ldots)$ be a sequence of compact intervals of $\mathbb{R}^{+}$such that $J_{k} \subset J_{k+1}$ and $\cup_{k} J_{k}=$ $\mathbb{R}^{+}$. Consider $s, t \in J_{k}$, we obtain

$$
\left\|\varphi_{n}(t)-\varphi_{n}(s)\right\|_{E} \leq C_{3}|t-s|
$$

which gives the equicontinuity of $\left\{\varphi_{n}(t)\right\}_{n=1}^{\infty}$ in $C\left(J_{k}, E\right)$. Equation (5.12) implies that, for fixed $t,\left\{\varphi_{n}(t)\right\}_{n=1}^{\infty}$ is uniformly bounded in $E$, therefore there exists a subsequence of $\left\{\varphi_{n}(t)\right\}_{n=1}^{\infty}$, still denoted by $\left\{\varphi_{n}(t)\right\}_{n=1}^{\infty}$, and $\tilde{\varphi}_{t} \in E$ such that

$$
\varphi_{n}(t) \longrightarrow \tilde{\varphi}_{t}, \quad \text { weakly in } E \text { as } n \longrightarrow \infty
$$

If we use the same method used to prove [19, Lemma 3.2], we can show that the weak convergence, here, is a strong one, that is, for all $t \in J_{k},\left\{\varphi_{n}(t)\right\}_{n=1}^{\infty}$ is precompact in $E$. By Ascoli's theorem, there exists a subsequence $\left\{\varphi_{n_{1}}(t)\right\}$ of $\left\{\varphi_{n}(t)\right\}$ and $\varphi_{t} \in C\left(J_{1}, E\right)$ such that $\varphi_{n_{1}}(t)$ converges to $\varphi_{t}$ in $C\left(J_{1}, E\right)$. Again by Ascoli's theorem and induction, there exists a subsequence $\left\{\varphi_{n_{k+1}}(t)\right\}$ of $\left\{\varphi_{n_{k}}(t)\right\}$ such that $\varphi_{n_{k+1}}(t)$ converges to $\varphi_{t}$ in $C\left(J_{k+1}, E\right)$. Taking the diagonal subsequence in the usual way, there exists a subsequence $\left\{\varphi_{n_{k}}(t)\right\}$ of $\left\{\varphi_{n}(t)\right\}$, still denoted by $\left\{\varphi_{n}(t)\right\}_{n=1}^{\infty}$, and $\varphi(t) \in C\left(\mathbb{R}^{+}, E\right)$ such that

$$
\varphi_{n}(t) \longrightarrow \varphi(t) \quad \text { in } C(J, E) \text { as } n \longrightarrow \infty \text { for any compact set } J \subset \mathbb{R}^{+}
$$

By (5.12), there exists a constant $C_{4}=C_{4}\left(r_{0}\right)$ such that for $\varphi(t)=(u(t), v(t))^{T}=\left(\left(u_{i}(t)\right)\right.$, $\left.\left(v_{i}(t)\right)\right)_{i \in \mathbb{Z}}^{T} \in E, v(t)=\dot{u}(t)+\varepsilon u(t)$

$$
\|\varphi(t)\|_{E}=\left(\|B u\|^{2}+\lambda\|u\|^{2}+\|v\|^{2}\right)^{1 / 2} \leq C_{4}, \quad \forall t \in \mathbb{R}^{+} .
$$

Here we prove that $\varphi(t) \in \mathscr{B}$. By (5.16),

$$
\dot{\varphi}_{n}(t) \longrightarrow \dot{\varphi}(t) \quad \text { weakly star in } L^{\infty}\left(\mathbb{R}^{+}, E\right) \text { as } n \longrightarrow \infty \text {. }
$$

Let $i \in \mathbb{Z}$ and $n \geq|i|$. Since $\varphi_{n}(t)=\left(\left(u_{n, i}(t)\right),\left(v_{n, i}(t)\right)\right)_{|i| \leq n}^{T} \in \widetilde{E}$ is the solution of (5.9), it follows that for all $t \in \mathbb{R}^{+}$and $\|i\| \leq n-2$

$$
\ddot{u}_{n, i}+\delta \dot{u}_{n, i}+\alpha\left(A u_{n}\right)_{i}+\beta\left(B u_{n}\right)_{i}+\lambda u_{n, i}-\frac{1}{3} k\left(D\left(D^{*} u_{n}\right)^{3}\right)_{i}=f_{i}
$$


Therefore for all $\psi \in C_{0}^{\infty}(J)$, we obtain

$$
\begin{gathered}
\int_{J} \ddot{u}_{n, i} \psi(t) d t+\delta \int_{J} \dot{u}_{n, i} \psi(t) d t+\alpha \int_{J}\left(A u_{n}\right)_{i} \psi(t) d t+\beta \int_{J}\left(B u_{n}\right)_{i} \psi(t) d t \\
+\lambda \int_{J} u_{n, i} \psi(t) d t-\frac{1}{3} k \int_{J}\left(D\left(D^{*} u_{n}\right)^{3}\right)_{i} \psi(t) d t=\int_{J} f_{i} \psi(t) d t
\end{gathered}
$$

It is seen that

$$
\begin{aligned}
\left|\int_{J}\left(D\left(D^{*} u_{n}\right)^{3}\right)_{i} \psi(t) d t-\int_{J}\left(D\left(D^{*} u\right)^{3}\right)_{i} \psi(t) d t\right| \\
\quad \leq \sup _{t \in J}\left|\left(D\left(\left(D^{*} u_{n}\right)^{3}-\left(D^{*} u\right)^{3}\right)\right)_{i}\right| \int_{J}|\psi(t)| d t .
\end{aligned}
$$

Using (2.13), (5.12), and (5.20), there exists a constant $C_{5}=C_{5}\left(r_{0}\right)$ such that

$$
\begin{aligned}
\left|\left(D\left(\left(D^{*} u_{n}\right)^{3}-\left(D^{*} u\right)^{3}\right)\right)_{i}\right| & \leq 4\left|\left(\left(D^{*} u_{n}\right)^{3}-\left(D^{*} u\right)^{3}\right)_{i}\right| \\
& \leq 9\left|\left(D^{*}\left(u_{n}-u\right)\right)_{i}\right|^{2}\left|\left(D^{*} u_{n}\right)_{i}^{2}+\left(D^{*} u\right)_{i}^{2}\right|^{2} \\
& \leq C_{5}\left|u_{n, i}-u_{i}\right|^{2},
\end{aligned}
$$

and from (5.19), as $n \rightarrow \infty$,

$$
\sup _{t \in J}\left|u_{n, i}-u_{i}\right| \longrightarrow 0
$$

That as $n \rightarrow \infty$,

$$
\left|\int_{J}\left(D\left(D^{*} u_{n}\right)^{3}\right)_{i} \psi(t) d t-\int_{J}\left(D\left(D^{*} u\right)^{3}\right)_{i} \psi(t) d t\right| \longrightarrow 0 .
$$

Using (5.21), (5.22), (5.23), (5.26), and (5.27), it follows that for all $t \in J$,

$$
\ddot{u}_{i}+\delta \dot{u}_{i}+\alpha(A u)_{i}+\beta(B u)_{i}+\lambda u_{i}-\frac{1}{3} k\left(D\left(D^{*} u\right)^{3}\right)_{i}=f_{i} .
$$

But $J$ is arbitrary, thus, (5.28), holds for all $t \in \mathbb{R}^{+}$, which means that $\varphi(t)$ is a solution of (2.5) and (2.6). From (5.20), it follows that $\varphi(t)$ is bounded for $t \in \mathbb{R}^{+}$, that $\varphi(t) \in \mathscr{B}$, therefore $\varphi_{n}(0) \rightarrow \varphi(0) \in \mathscr{B}$, and the proof is completed.

Now we are ready to represent the main result of this section.

Theorem 5.3. If $f \in l^{2},(1.7)$, and (2.7), are satisfied, then

$$
\lim _{n \rightarrow \infty} d_{E}\left(\mathscr{B}_{n}, \mathscr{B}\right)=0
$$

where $d_{E}\left(\mathscr{B}_{n}, \mathscr{B}\right)=\sup _{a \in \mathscr{B}_{n}} \inf _{b \in \mathscr{B}}\|a-b\|_{E}$. 
Proof. We argue by contradiction. If the conclusion is not true, then there exists a sequence $\varphi_{n_{k}} \in \mathscr{B}_{n_{k}}$ and a constant $K>0$ such that

$$
d_{E}\left(\varphi_{n_{k}}, \mathscr{B}\right) \geq K>0 .
$$

However, by Lemma 5.2, we know that there exists a subsequence $\varphi_{n_{k_{m}}}$ of $\varphi_{n_{k}}$ such that

$$
d_{E}\left(\varphi_{n_{k_{m}}}, \mathscr{B}\right) \longrightarrow 0,
$$

which contradicts (5.30). The proof is completed.

Theorem 5.3 shows that the global attractor $\mathscr{B}$ of the lattice dynamical system (2.8) is upper semicontinuous with respect to the (cut-off) approximate finite-dimension dynamical system (5.9).

\section{References}

[1] V. S. Afraimovich, S.-N. Chow, and J. K. Hale, Synchronization in lattices of coupled oscillators, Phys. D 103 (1997), no. 1-4, 442-451.

[2] V. S. Afraimovich and V. I. Nekorkin, Chaos of traveling waves in a discrete chain of diffusively coupled maps, Internat. J. Bifur. Chaos Appl. Sci. Engrg. 4 (1994), no. 3, 631-637.

[3] P. W. Bates and A. Chmaj, A discrete convolution model for phase transitions, Arch. Ration. Mech. Anal. 150 (1999), no. 4, 281-305.

[4] P. W. Bates, K. Lu, and B. Wang, Attractors for lattice dynamical systems, Internat. J. Bifur. Chaos Appl. Sci. Engrg. 11 (2001), no. 1, 143-153.

[5] J. Bell, Some threshold results for models of myelinated nerves, Math. Biosci. 54 (1981), no. 3-4, 181-190.

[6] J. Bell and C. Cosner, Threshold behavior and propagation for nonlinear differential-difference systems motivated by modeling myelinated axons, Quart. Appl. Math. 42 (1984), no. 1, 114.

[7] S.-N. Chow and J. Mallet-Paret, Pattern formation and spatial chaos in lattice dynamical systems. I, IEEE Trans. Circuits Systems I Fund. Theory Appl. 42 (1995), no. 10, 746-751.

[8] _ Pattern formation and spatial chaos in lattice dynamical systems. II, IEEE Trans. Circuits Systems I Fund. Theory Appl. 42 (1995), no. 10, 752-756.

[9] S.-N. Chow, J. Mallet-Paret, and W. Shen, Traveling waves in lattice dynamical systems, J. Differential Equations 149 (1998), no. 2, 248-291.

[10] S.-N. Chow, J. Mallet-Paret, and E. S. Van Vleck, Pattern formation and spatial chaos in spatially discrete evolution equations, Random Comput. Dynam. 4 (1996), no. 2-3, 109-178.

[11] R. Dogaru and L. O. Chua, Edge of chaos and local activity domain of FitzHugh-Nagumo equation, Internat. J. Bifur. Chaos Appl. Sci. Engrg. 8 (1998), no. 2, 211-257.

[12] J. P. Keener, Propagation and its failure in coupled systems of discrete excitable cells, SIAM J. Appl. Math. 47 (1987), no. 3, 556-572.

[13] The effects of discrete gap junction coupling on propagation in myocardium, J. Theoret. Biol. 148 (1991), no. 1, 49-82.

[14] N. Rashevsky, Mathematical Biophysics: Physico-Mathematical Foundations of Biology, 3rd revised ed. 2 vols, Dover Publications, New York, 1960.

[15] AC. Scott, Analysis of a myelinated nerve model, Bull. Math. Biophys. 26 (1964), 247-254.

[16] W. Shen, Lifted lattices, hyperbolic structures, and topological disorders in coupled map lattices, SIAM J. Appl. Math. 56 (1996), no. 5, 1379-1399.

[17] S. Zhou, Dimension of the global attractor for damped nonlinear wave equations, Proc. Amer. Math. Soc. 127 (1999), no. 12, 3623-3631. 
[18] Attractors for second order lattice dynamical systems, J. Differential Equations 179 (2002), no. 2, 605-624.

[19] Attractors and approximations for lattice dynamical systems, J. Differential Equations 200 (2004), no. 2, 342-368.

[20] B. Zinner, Existence of traveling wavefront solutions for the discrete Nagumo equation, J. Differential Equations 96 (1992), no. 1, 1-27.

Ahmed Y. Abdallah: Department of Mathematics, University of Jordan, Amman 11942, Jordan E-mail address: farah@ju.edu.jo 


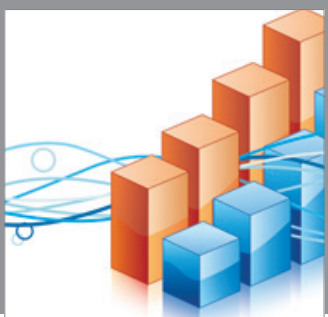

Advances in

Operations Research

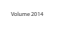

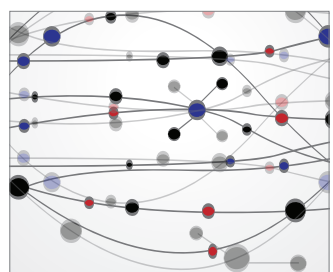

\section{The Scientific} World Journal
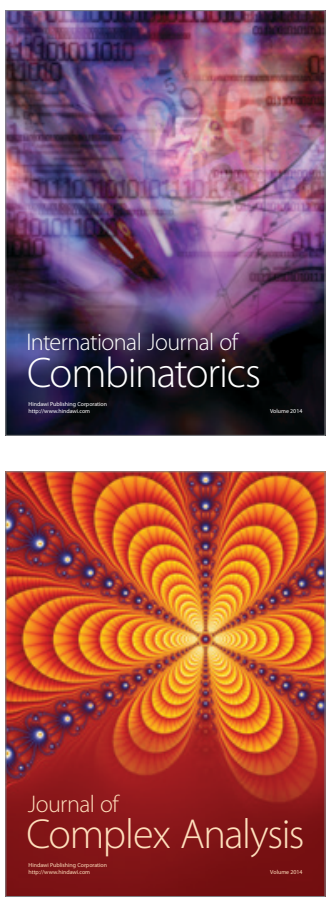

International Journal of

Mathematics and

Mathematical

Sciences
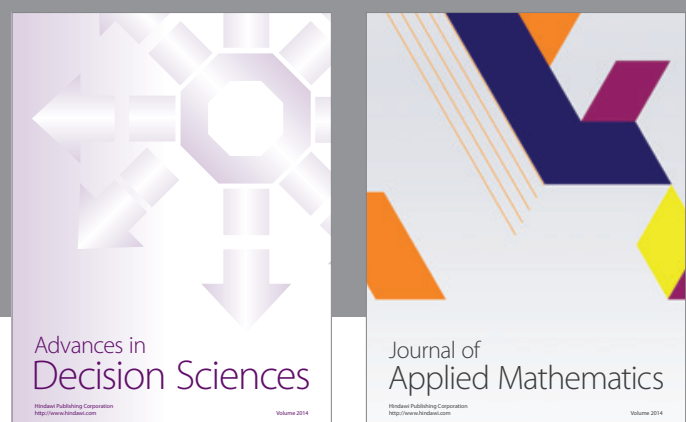

Journal of

Applied Mathematics
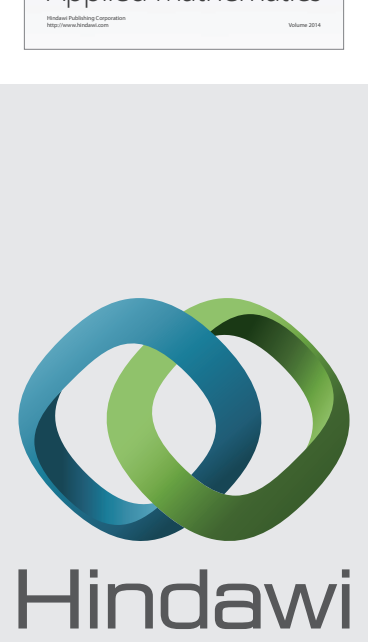

Submit your manuscripts at http://www.hindawi.com
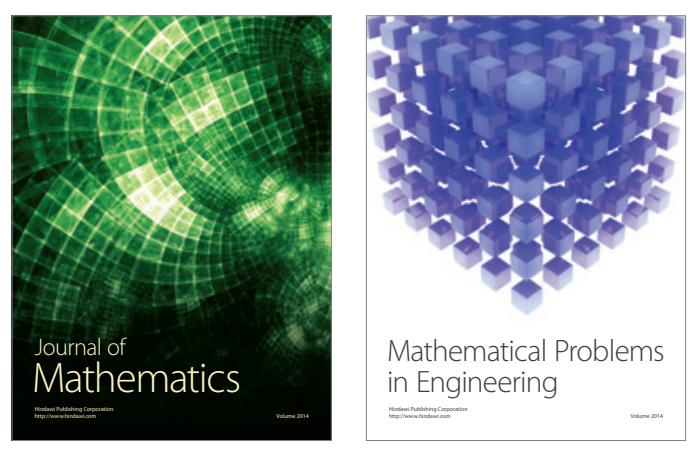

Mathematical Problems in Engineering
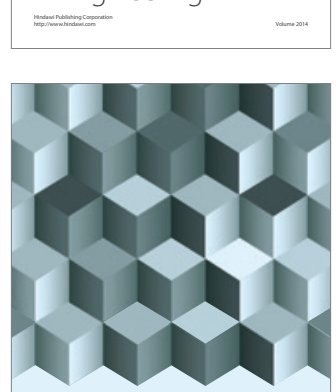

Journal of

Function Spaces
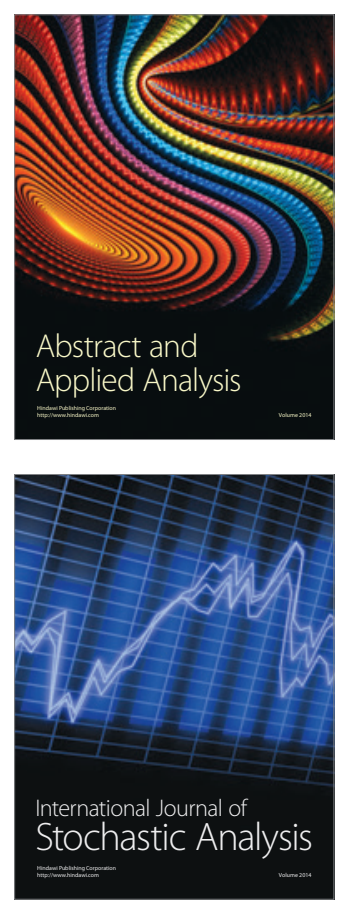

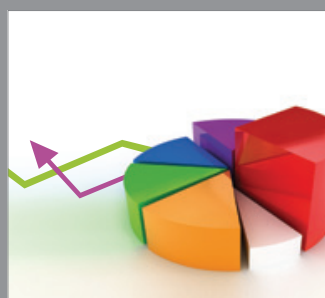

ournal of

Probability and Statistics

Promensencen
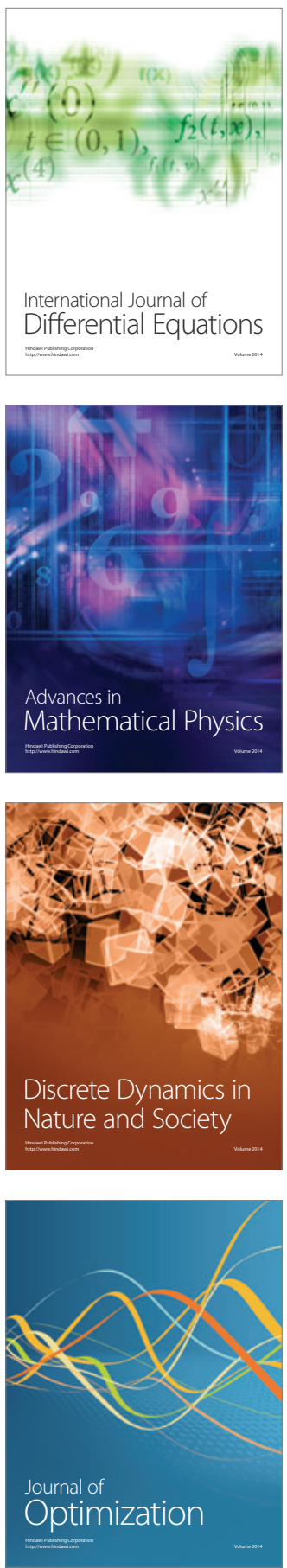